\title{
Homomorfisma Modul Deret Pangkat Tergeneralisasi Miring
}

\author{
AhMAD FAISOL $^{1}$, Fitriani $^{2}$ \\ Jurusan Matematika, Fakultas MIPA, Universitas Lampung, \\ Jl. Prof. Dr. Sumantri Brojonegoro No.1 Bandar Lampung 35145 \\ Email: ${ }^{1}$ ahmadfasiol@fmipa.unila.ac.id, ${ }^{2}$ fitriani.1984@fmipa.unila.ac.id
}

\begin{abstract}
Abstrak
Diberikan sebarang ring komutatif $R$ dengan elemen satuan, monoid terurut tegas $(S, \leq)$, homomorfisma monoid $\omega: S \rightarrow \operatorname{End}(R)$, submonoid $S_{1}, S_{2} \subseteq S$ yang masing-masing dilengkapi urutan $\leq_{1}, \leq_{2}$ yang coarser terhadap urutan $\leq$ pada $S$, dan modul $M_{1}, M_{2}$ atas $R$. Pada penelitian ini, dikonstruksi modul deret pangkat tergeneralisasi miring $M_{1}\left[\left[S_{1}, \leq_{1}, \omega\right]\right]$ dan $M_{2}\left[\left[S_{2}, \leq_{2}, \omega\right]\right]$ atas ring deret pangkat tergeneralisasi miring $R[[S, \leq, \omega]]$. Selain itu, dibuktikan pemetaan $\tau$ dari $M_{1}\left[\left[S_{1}, \leq_{1}, \omega\right]\right]$ ke $M_{2}\left[\left[S_{2}, \leq_{2}, \omega\right]\right]$ dengan $\tau\left(\alpha_{1}\right)=\gamma \circ \alpha_{1} \circ \delta^{-1}$ merupakan $R[[S, \leq, \omega]]$-homomorfisma modul dengan mensyaratkan $f\left(\delta^{-1}(u)\right)=f(u)$ dan $\omega_{\delta^{-1}(v)}=\omega_{v}$ untuk setiap $u, v \in S_{2}$ dan $f \in R[[S, \leq, \omega]]$.

Kata kunci: monoid terurut tegas, homomorfisma ring, ring deret pangkat tergeneralisasi miring, homomorfisma modul, modul deret pangkat tergeneralisasi miring.
\end{abstract}

\begin{abstract}
Let $R$ be a commutative ring with identity element, $(S, \leq)$ a strictly ordered monoid, $\omega: S \rightarrow$ End $(R)$ a monoid homomorphism, $S_{1}, S_{2} \subseteq S$ are submonoids with order $\leq_{1}, \leq_{2}$ that coarser to $\leq$ on $S$, and $M_{1}, M_{2}$ are modules over $R$. In this research, we define the skew generalized power series modules $M_{1}\left[\left[S_{1}, \leq_{1}, \omega\right]\right]$ and $M_{2}\left[\left[S_{2}, \leq_{2}, \omega\right]\right]$ over the skew generalized power series rings $R[[S, \leq, \omega]]$. Furthermore, we prove that the map $\tau$ from $M_{1}\left[\left[S_{1}, \leq_{1}, \omega\right]\right]$ to $M_{2}\left[\left[S_{2}, \leq_{2}, \omega\right]\right]$ with $\tau\left(\alpha_{1}\right)=\gamma \circ \alpha_{1} \circ$ $\delta^{-1}$ is an $R[[S, \leq, \omega]]$-homomorphism module by giving the sufficient conditions $f\left(\delta^{-1}(u)\right)=f(u)$ and $\omega_{\delta^{-1}(v)}=\omega_{v}$ for every $u, v \in S_{2}$ and $f \in R[[S, \leq, \omega]]$.

Keywords: strictly ordered monoid, ring homomorphism, skew generalized power series rings, homomorphism of modules, skew generalized power series modules.
\end{abstract}

\section{Pendahuluan}

Di dalam [1], dijelaskan bahwa ring adalah sebarang himpunan tak kosong yang dilengkapi dua operasi biner yang memenuhi beberapa aksioma. Pada tahun 1990, Ribenboim [2] mengkonstruksi Ring Deret Pangkat Tergeneralisasi (RDPT) $\left[\left[R^{S, \leq}\right]\right]$ yang merupakan perumuman dari ring semigrup $R[S][3]$, ring polinomial $R[X]$ dan ring deret pangkat $R[[X]]$ [4], dengan cara mensyaratkan $\operatorname{supp}(f)$ bersifat Artin dan narrow.

2000 Mathematics Subject Classification: 06F25, 13C05 Received: 17-07-2021, Revised: 25-11-2021; Accepted: 7-12-2021. 
Himpunan terurut parsial [5] $(S, \leq)$ dikatakan Artin, jika setiap barisan terurut tegas dari elemen $S$ berhingga, sedangkan dikatakan narrow jika setiap subhimpunan $S$ yang terurut trivial berhingga [6]. Sifat-sifat yang ada pada RDPT $\left[\left[R^{S, \leq}\right]\right]$ dapat dilihat pada [7][8][9][10][11]. Suatu ring dapat dipandang sebagai modul atas dirinya sendiri. Pada tahun 2001, Varadarajan [12] menkonstruksi Modul Deret Pangkat Tergeneralisasi (MDPT) $M[[S, \leq]]$, yang merupakan modul atas RDPT $\left[\left[R^{S, \leq}\right]\right]$. Beberapa penelitian terkait struktur MDPT $M[[S, \leq]]$ dapat dilihat pada [13][14][15][16][17]. Pada tahun 2008, Mazurek dan Ziembowski memperumum struktur RDPT $\left[\left[R^{S, \leq}\right]\right]$ dengan menggunakan homomorfisma monoid $\omega: S \rightarrow \operatorname{End}(R)[18]$. Hasil perumuman ini disebut Ring Deret Pangkat Tergeneralisasi Miring (RDPTM) $R[[S, \leq, \omega]]$, yang sifat-sifatnya dapat dilihat pada [19][20][21][22][23]. Selain itu, beberapa penelitian lain terkait RDPTM $R[[S, \leq, \omega]]$ juga dapat dilihat pada [24][25][26][27][28][29][30].

Berdasarkan struktur MDPT $M[[S, \leq]]$ yang telah dikonstruksi oleh Varadarajan, pada penelitian ini akan dikonstruksi modul atas RDPTM $R[[S, \leq, \omega]]$ yang selanjutnya disebut Modul Deret Pangkat Tergeneralisasi Miring (MDPTM) $M_{1}\left[\left[S_{1}, \leq_{1}, \omega\right]\right]$ dan $M\left[\left[S_{2}, \leq_{2}, \omega\right]\right]$, dengan $R$ adalah sebarang ring komutatif dengan elemen satuan, $(S, \leq)$ monoid terurut tegas, $\omega: S \rightarrow \operatorname{End}(R)$ suatu homomorfisma monoid, $S_{1}, S_{2} \subseteq S$ merupakan submonoid dengan urutan $\leq_{1}, \leq_{2}$ yang coarser terhadap $\leq$, dan $M_{1}, M_{2}$ adalah modul atas $R$. Hasil utama dari penelitian ini adalah didefinisikannya pemetaan $\tau$ dari $M_{1}\left[\left[S_{1}, \leq_{1}, \omega\right]\right]$ ke $M_{2}\left[\left[S_{2}, \leq_{2}, \omega\right]\right]$ dengan $\tau\left(\alpha_{1}\right)=\gamma \circ \alpha_{1} \circ \delta^{-1}$ yang merupakan $R[[S, \leq, \omega]]$-homomorfisma modul dengan mensyaratkan $f\left(\delta^{-1}(u)\right)=f(u)$ dan $\omega_{\delta^{-1}(v)}=\omega_{v}$ untuk setiap $u, v \in S_{2}$ dan $f \in R[[S, \leq, \omega]]$.

\section{Metode Penelitian}

Penelitian ini dilakukan berdasarkan studi literatur berupa buku dan jurnal imiah yang terkait dengan konsep modul atas ring, himpunan terurut yang bersifat Artin dan narrow, ring deret pangkat tergeneralisasi (RDPT), modul deret pangkat tergeneralisasi (MDPT), dan ring deret pangkat tergeneralisasi miring (RDPTM). Adapun langkah-langkah yang digunakan yaitu; mengkonstruksi MDPTM $M_{1}\left[\left[S_{1}, \leq_{1}, \omega\right]\right]$ dan $M_{2}\left[\left[S_{2}, \leq_{2}, \omega\right]\right]$ atas RDPTM $R[[S, \leq, \omega]]$, memberikan homomorfisma monoid tegas $\delta: S_{1} \rightarrow S_{2}$ dan $R$-homomorfisma modul $\gamma: M_{1} \rightarrow$ $M_{2}$, mendefinisikan pemetaan $\tau$ dari $M_{1}\left[\left[S_{1}, \leq_{1}, \omega\right]\right]$ ke $M_{2}\left[\left[S_{2}, \leq_{2}, \omega\right]\right]$ dengan $\tau\left(\alpha_{1}\right)=\gamma \circ$ $\alpha_{1} \circ \delta^{-1}$, memberikan syarat $f\left(\delta^{-1}(u)\right)=f(u)$ dan $\omega_{\delta^{-1}(v)}=\omega_{v}$ untuk setiap $u, v \in S_{2}$ dan $f \in R[[S, \leq, \omega]]$, dan terakhir membuktikan pemetaan $\tau$ merupakan $R[[S, \leq, \omega]]$-homomorfisma modul.

\section{Hasil Dan PEMBahasan}

Pada bagian ini dibahas tentang modul atas RDPTM dan homomorfisma modul atas RDPTM yang dikonstruksi berdasarkan struktur RDPTM $R[[S, \leq, \omega]]$ yang telah dikonstruksi oleh Mazurek dan Ziemboeski [18].

Pada penelitian ini, $R$ melambangkan sebarang ring komutatif dengan elemen satuan, $\operatorname{End}(R)$ adalah himpunan semua endomorfisma ring $R$ yang merupakan monoid terhadap operasi komposisi fungsi. $(S, \leq)$ disebut monoid terurut tegas artinya $S$ merupakan monoid terhadap operasi penjumlahan sekaligus $(S, \leq)$ merupakan himpunan terurut terhadap relasi urutan parsial $\leq$ yang tegas. Himpunan terurut $(S, \leq)$ dikatakan Artin, jika setiap barisan terurut tegas dari elemen $S$ berhingga, sedangkan dikatakan narrow jika setiap subhimpunan $S$ yang terurut trivial berhingga [6].

Diberikan homomorfisma monoid $\omega: S \rightarrow \operatorname{End}(R)$ dengan $\omega(s)=\omega_{s}$ untuk setiap $s \in S$. Oleh karena itu, untuk setiap $s, t \in S$ berlaku $\omega_{s+t}=\omega(s+t)=\omega(s) \circ \omega(t)=$ $\omega_{s} \circ \omega_{t}$. Jika $0 \in S$ elemen identitas di $S$, maka $\omega_{0}$ merupakan elemen identitas di $\operatorname{End}(R)$. Selanjutnya, didefinisikan himpunan $R[[S, \leq, \omega]]=\{f: S \rightarrow R \mid \operatorname{supp}(f)$ Artin dan narrow $\}$ dengan $\operatorname{supp}(f)=\{s \in S \mid f(s) \neq 0\} \subseteq(S, \leq)$. Untuk setiap $f, g \in R[[S, \leq, \omega]]$ dan $s \in S$, terhadap operasi

$$
(f+g)(s)=f(s)+g(s)
$$


dan

$$
(f g)(s)=\sum_{x+y=s} f(x) \omega_{x}(g(y)),
$$

$R[[S, \leq, \omega]]$ merupakan ring [18]. Ring ini disebut Ring Deret Pangkat Tergeneralisasi Miring (RDPTM).

Untuk sebarang $r \in R$ dan $s \in S$, didefinisikan pemetaan $c_{r}, f_{s} \in R[[S, \leq, \omega]]$ sebagai berikut:

$$
c_{r}(u)= \begin{cases}r & ; \text { jika } u=0 \\ 0 & ; \text { jika } u \neq 0\end{cases}
$$

and

$$
f_{s}(u)= \begin{cases}1 & ; \text { jika } u=s \\ 0 & ; \text { jika } u \neq s\end{cases}
$$

untuk setiap $u \in S$.

Berdasarkan (3), untuk setiap $u \in S$ berlaku

$$
c_{r_{1}+r_{2}}(u)=\left\{\begin{array}{ll}
r_{1}+r_{2} & ; \text { jika } u=0 \\
0 & ; \text { jika } u \neq 0,
\end{array}=c_{r_{1}}(u)+c_{r_{2}}(u),\right.
$$

dan

$$
c_{r_{1} r_{2}}(u)=\left\{\begin{array}{ll}
r_{1} r_{2} & ; \text { jika } u=0 \\
0 & ; \text { jika } u \neq 0,
\end{array}=c_{r_{1}}(u) c_{r_{2}}(u) .\right.
$$

Dengan kata lain, untuk sebarang $c_{r_{1}}, c_{r_{2}} \in R[[S, \leq, \omega]]$ berlaku

$$
c_{r_{1}+r_{2}}=c_{r_{1}}+c_{r_{2}} \text {, }
$$

dan

$$
c_{r_{1} r_{2}}=c_{r_{1}} c_{r_{2}},
$$

untuk setiap $r_{1}, r_{2} \in R$.

Selanjutnya, $r \mapsto c_{r}$ merupakan penyisipan ring (ring embedding) dari $R$ ke $R[[S, \leq, \omega]$, dan $s \mapsto f_{s}$ merupakan penyisipan monoid (monoid embedding) dari $S$ ke monoid multiplikatif (operasi komposisi) $R[[S, \leq, \omega]]$.

Proposisi 3.1. Diberikan $R D P T M R[[S, \leq, \omega]]$. Pemetaan $\varphi: R \rightarrow R[[S, \leq, \omega]]$ yang didefinisikan oleh $\varphi(r)=c_{r}$ untuk setiap $r \in R$, merupakan isomorfisma ring.

BukTi. Akan ditunjukkan $\varphi$ merupakan homomorfisma ring yang injektif dan surjektif.

(i) Berdasarkan definisi $\varphi$, untuk setiap $r_{1}, r_{2} \in R, \varphi\left(r_{1}+r_{2}\right)=c_{r_{1}+r_{2}}$ dan $\varphi\left(r_{1} r_{2}\right)=c_{r_{1} r_{2}}$. Berdasarkan (5), $\varphi\left(r_{1}+r_{2}\right)=c_{r_{1}+r_{2}}=c_{r_{1}}+c_{r_{2}}$. Kemudian, berdasarkan definisi $\varphi$ diperoleh $\varphi\left(r_{1}+r_{2}\right)=c_{r_{1}+r_{2}}=c_{r_{1}}+c_{r_{2}}=\varphi\left(r_{1}\right)+\varphi\left(r_{2}\right)$. Berdasarkan $(6), \varphi\left(r_{1} r_{2}\right)=$ $c_{r_{1} r_{2}}=c_{r_{1}} c_{r_{2}}$. Kemudian, berdasarkan definisi $\varphi$ diperoleh $\varphi\left(r_{1} r_{2}\right)=c_{r_{1} r_{2}}=c_{r_{1}} c_{r_{2}}=$ $\varphi\left(r_{1}\right) \varphi\left(r_{2}\right)$. Dengan kata lain, terbukti bahwa $\varphi$ merupakan homomorfisma ring.

(ii) Jika $\varphi\left(r_{1}\right)=\varphi\left(r_{2}\right)$, maka $c_{r_{1}}=c_{r_{2}}$ untuk setiap $r_{1}, r_{2} \in R$. Dengan kata lain, $c_{r_{1}}(u)=$ $c_{r_{2}}(u)$ untuk setiap $u \in S$. Berdasarkan (3), diperoleh $r_{1}=r_{2}$. Oleh karena itu, terbukti $\varphi$ injektif.

(iii) Derdasarkan (3), jelas berlaku untuk setiap $c_{r} \in R[[S, \leq, \omega]]$, terdapat $r \in R$ sehingga $\varphi(r)=c_{r}$. Dengan kata lain terbukti $\varphi$ surjektif.

Dari $(i)-($ iii $)$ terbukti bahwa $\varphi$ merupakan isomorfisma ring.

Lemma berikut menunjukkan hubungan antara fungsi $f_{s}$ pada (4) dan fungsi $c_{r}$ pada (3) di RDPTM $R[[S, \leq, \omega]]$.

Proposisi 3.2. Diberikan RDPTM $R[[S, \leq, \omega]]$. Untuk sebarang $s \in S$ dan $r \in R$ berlaku $f_{s} c_{r}=c_{\omega_{s}(r)} f_{s}$ untuk setiap $c_{r}, f_{s} \in R[[S, \leq, \omega]]$. 
BukTi. Cukup ditunjukkan berlaku $\left(f_{s} c_{r}\right)(u)=\left(c_{\omega_{s}(r)} f_{s}\right)(u)$, untuk setiap $u \in S$.

(Kasus 1) Jika $u \neq 0$, maka berdasarkan operasi perkalian pada (2) diperoleh

$$
\begin{aligned}
\left(f_{s} c_{r}\right)(u) & =\sum_{x+y=u} f_{s}(x) \omega_{x}\left(c_{r}(y)\right) \\
& =\underbrace{f_{x}}_{x=0, y=u \quad f_{s}(0) \omega_{0}\left(c_{r}(u)\right)}+\underbrace{f_{s}(u) \omega_{u}\left(c_{r}(0)\right)}_{x, y=0}+\underbrace{0+0+0+\cdots}_{x \neq 0, y \neq 0} \\
& =f_{s}(0) c_{r}(u)+f_{s}(u) \omega_{u}(r) \\
& =f_{s}(0) .0+f_{s}(u) \omega_{u}(r) \\
& =f_{s}(u) \omega_{u}(r) \\
& = \begin{cases}\omega_{s}(r) & ; u=s \\
0 & ; u \neq s\end{cases}
\end{aligned}
$$

Di sisi lain,

$$
\begin{aligned}
\left(c_{\omega_{s}(r)} f_{s}\right)(u) & =\sum_{x+y=u} c_{\omega_{s}(r)}(x) \omega_{x}\left(f_{s}(y)\right) \\
& =\underbrace{c_{\omega_{s}(r)}(0) \omega_{0}\left(f_{s}(u)\right)}_{x=0, y=u}+\underbrace{c_{\omega_{s}(r)}(u) \omega_{u}\left(f_{s}(0)\right)}_{x=u, y=0}+\underbrace{0+0+0+\cdots}_{x \neq 0, y \neq 0} \\
& =\omega_{s}(r) f_{s}(u)+0 . \omega_{u}\left(f_{s}(0)\right) \\
& =\omega_{s}(r) f_{s}(u) \\
& = \begin{cases}\omega_{s}(r) & ; u=s \\
0 & ; u \neq s\end{cases}
\end{aligned}
$$

Jadi, terbukti $\left(f_{s} c_{r}\right)(u)=\left(c_{\omega_{s}(r)} f_{s}\right)(u)$ untuk $u \neq 0 \in S$.

(Kasus 2) Jika $u=0$, maka berdasarkan operasi perkalian pada (2) diperoleh

$$
\begin{aligned}
\left(f_{s} c_{r}\right)(0) & =\sum_{x+y=0} f_{s}(x) \omega_{x}\left(c_{r}(y)\right) \\
& =\underbrace{f_{s}(0) \omega_{0}\left(c_{r}(0)\right)}_{x=0, y=0}+\underbrace{f_{s}(x) \omega_{x}\left(c_{r}(-x)\right)}_{y=-x}+\underbrace{0+0+0+\cdots}_{\nexists x, y \in S \ni x+y=0} \\
& =f_{s}(0) c_{r}(0)+f_{s}(x) \omega_{x}\left(c_{r}(-x)\right) \\
& =f_{s}(0) \cdot r+f_{s}(x) \omega_{x}\left(c_{r}(-x)\right) \\
& =\left\{\begin{array}{ll}
r+f_{0}(x) \omega_{x}\left(c_{r}(-x)\right) \quad ; s=0 \\
f_{s}(x) \omega_{x}\left(c_{r}(-x)\right)
\end{array} ; s \neq 0\right. \\
& = \begin{cases}2 r & ; s=0 \text { dan } x=0 \\
r & ; s=0 \text { dan } x \neq 0 \\
0 & ; s \neq 0 \text { dan } x=s \\
0 & ; s \neq 0 \text { dan } x \neq s\end{cases}
\end{aligned}
$$


Di sisi lain,

$$
\begin{aligned}
& \left(c_{\omega_{s}(r)} f_{s}\right)(0)=\sum_{x+y=0} c_{\omega_{s}(r)}(x) \omega_{x}\left(f_{s}(y)\right) \\
& =\underbrace{c_{\omega_{s}(r)}(0) \omega_{0}\left(f_{s}(0)\right)}_{x=0, y=0}+\underbrace{c_{\omega_{s}(r)}(x) \omega_{x}\left(f_{s}(-x)\right)}_{y=-x}+\underbrace{0+0+0+\cdots}_{\nexists x, y \in S \ni x+y=0} \\
& =\omega_{s}(r) f_{s}(0)+c_{\omega_{s}(r)}(x) \omega_{x}\left(f_{s}(-x)\right) \\
& = \begin{cases}\omega_{0}(r)+c_{\omega_{0}(r)}(x) \omega_{x}\left(f_{0}(-x)\right) & ; s=0 \\
c_{\omega_{s}(r)}(x) \omega_{x}\left(f_{s}(-x)\right) & ; s \neq 0\end{cases} \\
& = \begin{cases}r+c_{r}(x) \omega_{x}\left(f_{0}(-x)\right) & ; s=0 \\
c_{\omega_{s}(r)}(x) \omega_{x}\left(f_{s}(-x)\right) & ; s \neq 0\end{cases} \\
& = \begin{cases}r+r \omega_{0}(1) & ; s=0 \text { dan } x=0 \\
r+0 . \omega_{x}\left(f_{0}(-x)\right) & ; s=0 \text { dan } x \neq 0 \\
c_{\omega_{s}(r)}(s) \omega_{s}(0) & ; s \neq 0 \text { dan } x=s \\
c_{\omega_{s}(r)}(x) \omega_{x}(0) & ; s \neq 0 \text { dan } x \neq s\end{cases} \\
& = \begin{cases}2 r & ; s=0 \text { dan } x=0 \\
r & ; s=0 \text { dan } x \neq 0 \\
0 & ; s \neq 0 \text { dan } x=s \\
0 & ; s \neq 0 \text { dan } x \neq s\end{cases}
\end{aligned}
$$

Jadi, terbukti $\left(f_{s} c_{r}\right)(u)=\left(c_{\omega_{s}(r)} f_{s}\right)(u)$ untuk $u=0 \in S$. Dengan kata lain, terbukti $\left(f_{s} c_{r}\right)(u)=\left(c_{\omega_{s}(r)} f_{s}\right)(u)$, untuk setiap $u \in S$. Akibatnya untuk sebarang $s \in S$ dan $r \in R$ terbukti bahwa $f_{s} c_{r}=c_{\omega_{s}(r)} f_{s}$ untuk setiap $f_{s}, c_{r} \in R[[S, \leq, \omega]]$.

Diberikan submonoid $S_{1}, S_{2} \subseteq S$ yang masing-masing dilengkapi dengan urutan $\leq_{1}$ dan $\leq_{2}$ yang coarser terhadap urutan $\leq$ pada $S$; yaitu untuk $i=1,2$, jika $s \leq_{i} t$, maka $s \leq t$ untuk setiap $s, t \in S_{i}$. Jika diberikan modul $M_{1}$ dan $M_{2}$ atas $\operatorname{ring} R$, maka dapat dikonstruksi modul $M_{1}\left[\left[S_{1}, \leq_{1}, \omega\right]\right]$ dan $M_{2}\left[\left[S_{2}, \leq_{2}, \omega\right]\right]$ atas RDPTM $R[[S, \leq, \omega]]$ sebagai berikut:

(1) Himpunan tak kosong $M_{1}\left[\left[S_{1}, \leq_{1}, \omega\right]\right]=\left\{\alpha_{1}: S_{1} \rightarrow M_{1} \mid \operatorname{supp}\left(\alpha_{1}\right)\right.$ Artin dan narrow $\}$ dengan $\operatorname{supp}\left(\alpha_{1}\right)=\left\{s \in S_{1} \mid \alpha_{1}(s) \neq 0\right\} \subseteq\left(S_{1}, \leq_{1}\right)$ yang dilengkapi dengan operasi penjumlahan dan perkalian skalar

$$
\left(\alpha_{1}+\beta_{1}\right)(x)=\alpha_{1}(x)+\beta_{1}(x)
$$

dan

$$
\left(\alpha_{1} f\right)(x)=\sum_{u+v=x} \alpha_{1}(u) \omega_{u}(f(v)),
$$

untuk setiap $f \in R[[S, \leq, \omega]], \alpha_{1}, \beta_{1} \in M_{1}\left[\left[S_{1}, \leq_{1}, \omega\right]\right]$, dan $x \in S_{1} \subseteq S$.

(2) Himpunan tak kosong $M_{2}\left[\left[S_{2}, \leq_{2}, \omega\right]\right]=\left\{\alpha_{2}: S_{2} \rightarrow M_{2} \mid \operatorname{supp}\left(\alpha_{2}\right)\right.$ Artin dan narrow $\}$ dengan $\operatorname{supp}\left(\alpha_{2}\right)=\left\{s \in S_{2} \mid \alpha_{2}(s) \neq 0\right\} \subseteq\left(S_{2}, \leq_{2}\right)$ yang dilengkapi dengan operasi biner

$$
\left(\alpha_{2}+\beta_{2}\right)(y)=\alpha_{2}(y)+\beta_{2}(y)
$$

dan

$$
\left(\alpha_{2} f\right)(y)=\sum_{p+q=y} \alpha_{2}(p) \omega_{p}(f(q)),
$$

untuk setiap $f \in R[[S, \leq, \omega]], \alpha_{2}, \beta_{2} \in M_{2}\left[\left[S_{2}, \leq_{2}, \omega\right]\right]$, dan $y \in S_{2} \subseteq S$.

Kedua modul ini disebut Modul Deret Pangkat Tergeneralisasi Miring (MDPTM). Untuk $i=1,2, s_{i} \in S_{i}, m_{i} \in M_{i}$, terdapat $d_{m_{i}}^{s_{i}} \in M_{i}\left[\left[S_{i}, \leq_{i}, \omega\right]\right]$ yang didefinisikan sebagai berikut. 


$$
d_{m_{i}}^{s_{i}}(x)= \begin{cases}m_{i} & ; x=s_{i} \\ 0 & ; x \neq s_{i}\end{cases}
$$

Dari persamaan (3) dan (7), untuk $i=1,2$ dan setiap $x \in S_{i} \subseteq S$ berlaku

$$
d_{m_{i}+n_{i}}^{s_{i}}(x)=\left\{\begin{array}{ll}
m_{i}+n_{i} & ; x=s_{i} \\
0 & ; x \neq s_{i}
\end{array}=d_{m_{i}}^{s_{i}}(x)+d_{n_{i}}^{s_{i}}(x),\right.
$$

dan

$$
d_{m_{i} r}^{s_{i}}(x)=\left\{\begin{array}{ll}
m_{i} r & ; x=s_{i} \\
0 & ; x \neq s_{i}
\end{array}=d_{m_{i}}^{s_{i}}(x) c_{r}(x) .\right.
$$
berlaku

Dengan kata lain, untuk $i=1,2$ dan sebarang $c_{r} \in R[[S, \leq, \omega]], d_{m_{i}}^{s_{i}}, d_{n_{i}}^{s_{i}} \in M_{i}\left[\left[S_{i}, \leq_{i}, \omega\right]\right]$

dan

$$
d_{m_{i}+n_{i}}^{s_{i}}=d_{m_{i}}^{s_{i}}+d_{n_{i}}^{s_{i}}
$$

untuk setiap $r \in R, m_{i}, n_{i} \in M_{i}$, dan $s_{i} \in S_{i}$.

$$
d_{m_{i} r}^{s_{i}}=d_{m_{i}}^{s_{i}} c_{r}
$$

Selanjutnya, untuk $i=1,2$ didefinisikan pemetaan $\sigma_{i}: M_{i} \rightarrow M_{i}\left[\left[S_{i}, \leq_{i}, \omega\right]\right]$ dengan $\sigma_{i}\left(m_{i}\right)=d_{m_{i}}^{s_{i}}$ untuk setiap $m_{i} \in M_{i}$ dan $s_{i} \in S_{i}$. Misalkan $\delta:\left(S_{1}, \leq_{1}\right) \rightarrow\left(S_{2}, \leq_{2}\right)$ adalah homomorfisma monoid tegas, yaitu homomorfisma monoid yang memenuhi; jika $s \leq_{1} t$, maka $\delta(s) \leq_{2} \delta(t)$ untuk setiap $s, t \in S_{1}$. Jika diberikan $R$-homomorfisma modul $\gamma: M_{1} \rightarrow M_{2}$, maka dapat didefinisikan pemetaan $\tau$ dari $M_{1}\left[\left[S_{1}, \leq_{1}, \omega\right]\right]$ ke $M_{2}\left[\left[S_{2}, \leq_{2}, \omega\right]\right]$ yang disajikan pada gambar berikut.

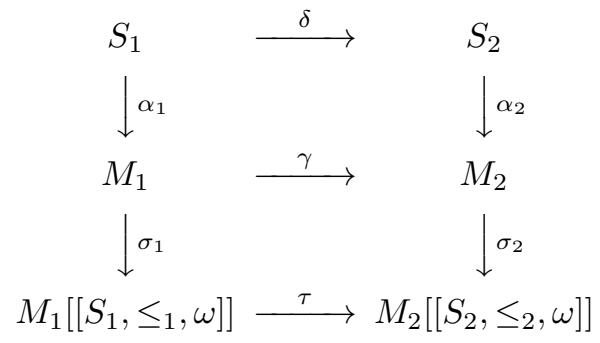

Gambar 1. Pemetaan $\tau$

Proposisi berikut menunjukkan bahwa pemetaan $\tau: M_{1}\left[\left[S_{1}, \leq_{1}, \omega\right]\right] \rightarrow M_{2}\left[\left[S_{2}, \leq_{2}, \omega\right]\right]$ yang didefinisikan oleh $\tau\left(\alpha_{1}\right)=\gamma \circ \alpha_{1} \circ \delta^{-1}$ untuk setiap $\alpha_{1} \in M_{1}\left[\left[S_{1}, \leq_{1}, \omega\right]\right]$ merupakan $R[[S, \leq, \omega]]$-homomorfisma modul.

Proposisi 3.3. Diberikan MDPTM $M_{1}\left[\left[S_{1}, \leq_{1}, \omega\right]\right], M_{2}\left[\left[S_{2}, \leq_{2}, \omega\right]\right]$ atas $R[[S, \leq, \omega]]$ dan $R$ homomorfisma modul $\gamma: M_{1} \rightarrow M_{2}$. Diberikan homomorfisma monoid tegas $\delta:\left(S_{1}, \leq_{1}\right) \rightarrow$ $\left(S_{2}, \leq_{2}\right)$ sehingga sebarang $T \subseteq S_{1}$ yang Artin dan narrow berlaku $\delta(T) \subseteq S_{2}$ juga Artin dan narrow. Didefinisikan pemetaan $\tau: M_{1}\left[\left[S_{1}, \leq_{1}, \omega\right]\right] \rightarrow M_{2}\left[\left[S_{2}, \leq_{2}, \omega\right]\right]$ dengan

$$
\tau\left(\alpha_{1}\right)=\gamma \circ \alpha_{1} \circ \delta^{-1} \text {, }
$$

untuk setiap $\alpha_{1} \in M_{1}\left[\left[S_{1}, \leq_{1}, \omega\right]\right]$. Jika untuk setiap $u, v \in S_{2} \subseteq S$ dan $f \in R[[S, \leq, \omega]]$, $f\left(\delta^{-1}(u)\right)=f(u)$ dan $\omega_{\delta^{-1}(v)}=\omega_{v}$, maka $\tau$ merupakan $R[[S, \leq, \omega]]$-homomorfisma modul.

BukTi. Untuk sebarang $\alpha_{1} \in M_{1}\left[\left[S_{1}, \leq_{1}, \omega\right]\right]$ Untuk sebarang $t \in S_{2}$,

$$
\begin{aligned}
\gamma \circ\left(\alpha_{1}+\beta_{1}\right) \circ \delta^{-1}(t) & =\gamma\left(\left(\alpha_{1}+\beta_{1}\right)\left(\delta^{-1}(t)\right)\right) \\
& =\gamma\left(\alpha_{1}\left(\delta^{-1}(t)\right)+\beta_{1}\left(\delta^{-1}(t)\right)\right) \\
& =\gamma \circ \alpha_{1} \circ \delta^{-1}(t)+\gamma \circ \beta_{1} \circ \delta^{-1}(t) .
\end{aligned}
$$


Dengan kata lain, berlaku

$$
\gamma \circ\left(\alpha_{1}+\beta_{1}\right) \circ \delta^{-1}=\left(\gamma \circ \alpha_{1} \circ \delta^{-1}\right)+\left(\gamma \circ \beta_{1} \circ \delta^{-1}\right) .
$$

Untuk sebarang $t \in S_{2}$

$$
\begin{aligned}
\gamma \circ\left(\alpha_{1} f\right) \circ \delta^{-1}(t) & =\gamma\left(\left(\alpha_{1} f\right)\left(\delta^{-1}(t)\right)\right) \\
& =\gamma\left(\sum_{x+y=\delta^{-1}(t)} \alpha_{1}(x) \omega_{x}(f(y))\right) \\
& =\sum_{x+y=\delta^{-1}(t)} \gamma\left(\alpha_{1}(x) \omega_{x}(f(y))\right) \\
& =\sum_{x+y=\delta^{-1}(t)} \gamma\left(\alpha_{1}(x)\right) \omega_{x}(f(y)) \quad ; x, y \in S_{1} \\
& =\sum_{u+v=t} \gamma\left(\alpha_{1}\left(\delta^{-1}(u)\right)\right) \omega_{\delta^{-1}(u)}\left(f\left(\delta^{-1}(v)\right)\right) ; \exists u, v \in S_{2} \ni \delta(x)=u ; \delta(y)=v \\
& =\sum_{u+v=t} \gamma\left(\alpha_{1}\left(\delta^{-1}(u)\right)\right) \omega_{u}(f(v)) \\
& =\sum_{u+v=t}\left(\gamma \circ \alpha_{1} \circ \delta^{-1}\right)(v) \omega_{u}(f(v)) \\
& =\left(\left(\gamma \circ \alpha_{1} \circ \delta^{-1}\right) f\right)(t) .
\end{aligned}
$$

Dengan kata lain, berlaku

$$
\gamma \circ\left(\alpha_{1} f\right) \circ \delta^{-1}=\left(\gamma \circ \alpha_{1} \circ \delta^{-1}\right) f .
$$

Selanjutnya akan ditunjukkan pemetaan $\tau$ merupakan homomorfisma, yaitu untuk setiap $\alpha_{1}, \beta_{1} \in M_{1}\left[\left[S_{1}, \leq_{1}, \omega\right]\right]$ dan $f \in R[[S, \leq, \omega]]$, berlaku $\tau\left(\alpha_{1}+\beta_{1}\right)=\tau\left(\alpha_{1}\right)+\tau\left(\beta_{1}\right)$ dan $\tau\left(\alpha_{1} f\right)=$ $\tau\left(\alpha_{1}\right) f$. Dari definisi pemetaan $\tau$, jelas $\tau\left(\alpha_{1}+\beta_{1}\right)=\gamma \circ\left(\alpha_{1}+\beta_{1}\right) \circ \delta^{-1}$. Berdasarkan (10) didapat $\gamma \circ\left(\alpha_{1}+\beta_{1}\right) \circ \delta^{-1}=\left(\gamma \circ \alpha_{1} \circ \delta^{-1}\right)+\left(\gamma \circ \beta_{1} \circ \delta^{-1}\right)$. Oleh karena itu, terbukti $\tau\left(\alpha_{1}+\beta_{1}\right)=\gamma \circ\left(\alpha_{1}+\beta_{1}\right) \circ \delta^{-1}=\left(\gamma \circ \alpha_{1} \circ \delta^{-1}\right)+\left(\gamma \circ \beta_{1} \circ \delta^{-1}\right)=\tau\left(\alpha_{1}\right)+\tau\left(\beta_{1}\right)$.

Di sisi lain, dari definisi pemetaan $\tau$, diketahui $\tau\left(\alpha_{1} f\right)=\gamma \circ\left(\alpha_{1} f\right) \circ \delta^{-1}$. Selanjutnya, berdasarkan (11) didapat $\gamma \circ\left(\alpha_{1} f\right) \circ \delta^{-1}=\left(\gamma \circ \alpha_{1} \circ \delta^{-1}\right) f$. Oleh karena itu, terbukti $\tau\left(\alpha_{1} f\right)=$ $\left(\gamma \circ \alpha_{1} \circ \delta^{-1}\right) f=\tau\left(\alpha_{1}\right) f$. Jadi, terbukti $\tau: M_{1}\left[\left[S_{1}, \leq_{1}, \omega\right]\right] \rightarrow M_{2}\left[\left[S_{2}, \leq_{2}, \omega\right]\right]$ merupakan $R[[S, \leq, \omega]]$-homomorfisma modul.

\section{Simpulan}

Dengan memberikan sebarang ring komutatif $R$ dengan elemen satuan, monoid terurut tegas $(S, \leq)$, homomorfisma monoid $\omega: S \rightarrow \operatorname{End}(R)$, submonoid $S_{1}, S_{2} \subseteq S$ yang masingmasing dilengkapi urutan $\leq_{1}, \leq_{2}$ yang coarser terhadap urutan $\leq$ pada $S$, dan modul $M_{1}, M_{2}$ atas $R$, maka dapat dikonstruksi MDPTM $M_{1}\left[\left[S_{1}, \leq_{1}, \omega\right]\right]$ dan $M_{2}\left[\left[S_{2}, \leq_{2}, \omega\right]\right]$ yang merupakan modul atas RDPTM $R[[S, \leq, \omega]]$.

Pemetaan $\tau$ dari MDPTM $M_{1}\left[\left[S_{1}, \leq_{1}, \omega\right]\right]$ ke MDPTM $M_{2}\left[\left[S_{2}, \leq_{2}, \omega\right]\right]$ dapat definisikan dengan terlebih dahulu memberikan homomorfisma monoid tegas $\delta: S_{1} \rightarrow S_{2}$ dan $R$-homomorfisma modul $\gamma: M_{1} \rightarrow M_{2}$, sehingga $\tau\left(\alpha_{1}\right)=\gamma \circ \alpha_{1} \circ \delta^{-1}$ untuk setiap $\alpha_{1} \in M_{1}\left[\left[S_{1}, \leq_{1}, \omega\right]\right]$. Dengan memberikan syarat $f\left(\delta^{-1}(u)\right)=f(u)$ dan $\omega_{\delta^{-1}(v)}=\omega_{v}$ untuk setiap $u, v \in S_{2}$ dan $f \in R[[S, \leq, \omega]]$, maka dapat dibuktikan bahwa $\tau$ merupakan $R[[S, \leq, \omega]]$-homomorfisma modul. 


\section{DAFtar Pustaka}

[1] Dummit, D.S., 2004, Abstract Algebra, John Wiley and Sons.

[2] Ribenboim, P., 1990, Generalized power series rings, in Lattice, Semigroups and Universal Algebra, Plenum Press, New York, pp 271-277.

[3] Gilmer, R., 1984, Commutative Semigroups Rings, University of Chicago Press, Chicago.

[4] Hungerford, T.W., 1974, Algebra, Springer-Verlag, New York.

[5] Adkins, W. A., Weintraub, S. H., 1992, Algebra: An Approach via Module Theory, Springer-Verlag, New York.

[6] Elliott, G.A., Ribenboim, P., 1990, Fields of Generalized Power Series, Arch. Math., 54:365-371.

[7] Ribenboim, P., 1991, Rings of Generalized Power Series: Nilpotent Elements, Abh. Math. Sem. Univ. Hambg., 61:15-33.

[8] Ribenboim, P., 1992, Noetherian Rings of Generalized Power Series, Journal of Pure and Applied Algebra, 79:293-312.

[9] Ribenboim, P., 1994, Rings of Generalized Power Series II: Units and Zero-Divisors, Journal of Algebra, 168:71-89.

[10] Ribenboim, P., 1995, Special Properties of Generalized Power Series, Journal of Algebra, 173:566-586.

[11] Ribenboim, P., 1997, Semisimple Rings and Von Neumann Regular Rings of Generalized Power Series, Journal of Algebra, 198:327-338.

[12] Varadarajan, K., 2001, Noetherian generalized power series rings and modules, Communications In Algebra 29(1):245-251.

[13] Faisol, A., Surodjo, B., Wahyuni, S., 2019, The Relation between Almost Noetherian Module, Almost Finitely Generated Module and T-Noetherian Module, The 2nd International Conference on Mathematics: Education, Theory, and Application (ICMETA 2018), J. Phys.: Conf. Ser. 1306., 012001.

[14] Faisol, A., Surodjo, B., Wahyuni, S., 2019, The Sufficient Conditions for $R[X]$-module $M[X]$ to be $S[X]$ Noetherian, European Journal of Mathematical Sciences, 5(1):1-13.

[15] Faisol, A., Surodjo, B., Wahyuni, S., 2019, T[[S]]-Noetherian Property on Generalized Power Series Modules, JP Journal of Algebra, Number Theory and Applications, 43(1):1-12.

[16] Pardede, W.A.P., Faisol, A., Fitriani, 2020, The $X[[S]]$-Sub-Exact Sequence of Generalized Power Series Rings, Al-Jabar J. Pendidik. Mat., 11(2):299-306.

[17] Faisol, A., Fitriani, Sifriyani, 2021, Determining the Noetherian Property of Generalized Power Series Modules by Using $X$-Sub-Exact Sequence, The 3rd International Conference on Applied Sciences Mathematics and Informatics (ICASMI 2020), J. Phys.: Conf. Ser. 1751., 012028.

[18] Mazurek, R., Ziembowski, M., 2008, On Von Neumann Regular Rings of Skew Generalized Power Series, Commun. Algebr., 36(5):1855-1868.

[19] Mazurek, R., Ziembowski, M., 2009, The ascending chain condition for principal left or right ideals of skew generalized power series rings, Journal of Algebra, 322(4):983-994.

[20] Mazurek, R., Ziembowski, M., 2010, Weak dimension and right distributivity of skew generalized power series rings, J. Math. Soc. Japan, 62(4):1093-1112.

[21] Mazurek, R., 2014, Rota-Baxter Operators on Skew Generalized Power Series Rings, J. Algebr. its Appl., 13(7):1-10

[22] Mazurek, R., 2015, Left Principally Quasi-Baer and Left APP-rings of Skew Generalized Power Series, $J$. Algebr. its Appl., 14(3):1-36.

[23] Mazurek, R., Paykan, K., 2017, Simplicity of skew generalized power series rings, New York J. Math., 23:1273-1293.

[24] Faisol, A., 2009, Homomorfisam Ring Deret Pangkat Teritlak Miring, J. Sains MIPA, 15(2):119-124.

[25] Faisol, A., 2010, Ideal Ring Deret Pangkat Teritlak Miring, in Prosiding Seminar Nasional Sains MIPA dan Aplikasinya 2010 (SNSMAP 10), pp 202-207.

[26] Faisol, A., 2013, Pembentukan Ring Faktor Pada Ring Deret Pangkat Teritlak Miring, in Prosiding Semirata FMIPA Universitas Lampung, pp 1-5.

[27] Faisol, A., 2014, Endomorfisma Rigid dan Compatible pada Ring Deret Pangkat Tergeneralisasi Miring, J. Matematika, 17(2):45-49.

[28] Faisol, A., Surodjo, B., dan Wahyuni, S., 2016, Modul Deret Pangkat Tergeneralisasi Skew T-Noether, in Prosiding Seminar Nasional Aljabar, Penerapan, dan Pembelajarannya, pp 95-100.

[29] Faisol, A., Surodjo, B., Wahyuni, S., 2018, The Impact of The Monoid Homomorphism on The Structure of Skew Generalized Power Series Rings, Far East Journal of Mathematical Sciences, 103(7):1215-12275.

[30] Faisol, A., Fitriani, 2019, The Sufficient Conditions for Skew Generalized Power Series Module $M[[S, \omega]]$ to be $T[[S, \omega]]$-Noetherian $R[[S, \omega]]$-module, Al-Jabar J. Pendidik. Mat., 10(2):285-292. 